\title{
GENERATING FUNCTION FOR GENERALIZED FUNCTION OF TWO VARIABLES
}

\author{
B. L. SHARMA AND R. F. A. ABIODUN
}

ABSTRACT. In this paper we obtain a generating function for a generalized function of $t$ wo variables. The result is very general in character and includes as particular cases some of the results recently given by Meijer [4], Carlitz [7] and Srivastava [5].

In a recent paper, Sharma [2] has defined the generalized function of two variables as follows:

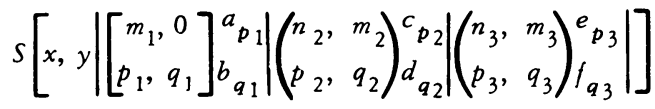

$$
\begin{gathered}
=\frac{1}{(2 \pi i)^{2}} \int_{c 1} \int_{c_{2}} \frac{\Pi_{j=1}^{m_{1}} \Gamma\left(a_{j}+s+t\right) \Pi_{j=1}^{m_{2}} \Gamma\left(1-c_{j}+s\right) \Pi_{j=1}^{n_{2}} \Gamma\left(d_{j}-s\right)}{\Pi_{j=m_{1}+1}^{p_{1}} \Gamma\left(1-a_{j}-s-t\right) \Pi_{j=1}^{q_{1}} \Gamma\left(b_{j}+s+t\right) \Pi_{j=m_{2}+1}^{p_{2}} \Gamma\left(c_{j}-s\right)} \\
\cdot \frac{\Pi_{j=1}^{m_{3}} \Gamma\left(1-e_{j}+t\right) \Pi_{j=1}^{n_{3}} \Gamma\left(f_{j}-t\right) x^{s} y^{t} d s d t}{\prod_{j=n_{2}+1}^{q_{2}} \Gamma\left(1-d_{j}+s\right) \Pi_{j=m_{3}+1}^{p_{3}} \Gamma\left(e_{j}-t\right) \Pi_{j=n_{3}+1}^{q_{3}} \Gamma\left(1-f_{j}+t\right)}
\end{gathered}
$$

where $c_{1}$ and $c_{2}$ are two suitable contours and positive integers $p_{1}, p_{2}$, $p_{3}, q_{1}, q_{2}, q_{3}, m_{1}, m_{2}, m_{3}, n_{2}$ and $n_{3}$ satisfy the following inequalities. $q_{2} \geq 1, q_{3} \geq 1, p_{1} \geq 0,0 \leq m_{1} \leq p_{1}, 0 \leq m_{2} \leq p_{2}, 0 \leq n_{2} \leq q_{2}, 0 \leq m_{3} \leq p_{3}$, $0 \leq n_{3} \leq q_{3}, p_{1}+p_{2} \leq q_{1}+q_{2}, p_{1}+p_{3} \leq q_{1}+q_{3}$. The values $x=0$ and $y=0$ are excluded.

We shall make use of the formula [6, Vol. I, Example 212, p. 126]

$$
\sum_{n=0}^{\infty}\left(\begin{array}{c}
a+(b+1) n \\
n
\end{array}\right) t^{n}=\frac{(1+v)^{a+1}}{1-b v}
$$

where $v$ is a function of $t$ given by $v(0)=0, v=t(1+v)^{b+1}$. We establish the following formula:

Received by the editors December 5, 1972.

AMS (MOS) subject classifications (1970). Primary 33A30, 33A35.

Key words and phrases. Generalized function of two variables, Appell function. 


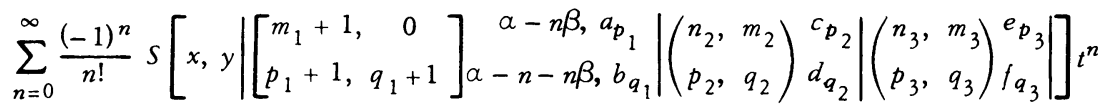

$$
\begin{aligned}
& =\frac{(1+v)^{1-a}}{1-\beta v} S\left[\frac{x}{1+v}, \frac{y}{1+v}\left|\left[\begin{array}{cc}
m_{1}, & 0 \\
p_{1}, & q_{1}
\end{array}\right]^{a_{p_{1}}} b_{q_{1}}\right|\left(\begin{array}{cc}
n_{2}, & m_{2} \\
p_{2}, & q_{2}
\end{array}\right)_{d_{q_{2}}}^{c_{p_{2}}}\left|\left(\begin{array}{cc}
n_{3}, & m_{3} \\
p_{3}, & q_{3}
\end{array}\right)_{f_{q_{3}}}^{e_{p_{3}}}\right|\right]
\end{aligned}
$$

where $v$ is a function of $t$ defined by $v(0)=0, v=t(1+v)^{\beta+1},|t|<1$, $|\arg x|<\left(m_{1}+m_{2}+n_{2}-1 / 2 p_{1}-1 / 2 q_{1}-1 / 2 p_{2}-1 / 2 q_{2}\right) \pi,|\arg y|<$ $\left(m_{1}+m_{3}+n_{3}-1 / 2 p_{1}-1 / 2 q_{1}-1 / 2 p_{2}-1 / 2 q_{2}\right) \pi, 2\left(m_{1}+m_{2}+n_{2}\right)>p_{1}+q_{1}+$ $p_{2}+q_{2}, 2\left(m_{1}+m_{3}+n_{3}\right)>p_{1}+q_{1}+p_{3}+q_{3}$.

To prove (3), substitute the contour integral (1) for the generalized function of two variables in the left side of (3), change the order of integration and summation, using (2) and (1); we get the right side of (3).

We mention some of the interesting particular cases of (3). Using the relation due to Sharma [3]

$$
s\left[x, y\left|\left[\begin{array}{ll}
0, & 0 \\
0, & 0
\end{array}\right]^{-}\right|\left(\begin{array}{ll}
n_{2}, & m_{2} \\
p_{2}, & q_{2}
\end{array}\right){ }_{d_{q_{2}}}^{c_{p_{2}}}\left|\left(\begin{array}{cc}
n_{3}, & m_{3} \\
p_{3}, & q_{3}
\end{array}\right)^{e_{p_{3}}} f_{q_{3}}\right|\right]
$$

$$
=G_{p_{2}, q_{2}}^{n_{2}, m_{2}}\left[x \mid \begin{array}{l}
c_{p_{2}} \\
d_{q_{2}}
\end{array}\right] G_{p_{3}, q_{3}}^{n_{3}, m_{3}}\left[y \mid \begin{array}{l}
e_{p_{3}} \\
f_{q_{3}}
\end{array}\right]
$$

in (3), we have

$$
\begin{aligned}
\sum_{n=0}^{\infty} \frac{(-1)^{n}}{n !} s\left[x,\left.y||\left[\begin{array}{ll}
1, & 0 \\
1, & 1
\end{array}\right]_{a-n-n \beta}^{\alpha-n \beta}\left|\left(\begin{array}{ll}
n_{2}, & m_{2} \\
p_{2} & q_{2}
\end{array}\right){ }_{d_{q_{2}}}^{c_{p_{2}}}\right|\left(\begin{array}{cc}
n_{3}, & m_{3} \\
p_{3}, & q_{3}
\end{array}\right)\right|_{f_{q_{3}}} ^{e_{p_{3}}} \mid\right] t^{n} \\
=\frac{(1+v)^{1-\alpha}}{1-\beta v} G_{p_{2}, q_{2}}^{n_{2}, m_{2}}\left[\frac{x}{1+v} \mid \begin{array}{c}
c_{p_{2}} \\
d_{q_{2}}
\end{array}\right] G_{p_{3}, q_{3}}^{n_{3}, m_{3}}\left[\left.\frac{y}{1+v}\right|_{f_{q_{3}}} ^{e_{p_{3}}}\right],
\end{aligned}
$$

provided that $v$ is a function of $t$ defined above and $|t|<1,2\left(m_{2}+n_{2}\right)>$ $p_{2}+q_{2}, 2\left(m_{3}+n_{3}\right)>p_{3}+q_{3},|\arg x|<\left(m_{2}+n_{2}-1 / 2 p_{2}-1 / 2 q_{2}\right) \pi,|\arg y|<$ $\left(m_{3}+n_{3}-1 / 2 p_{3}-1 / 2 q_{3}\right) \pi$. Further we use the relation due to Sharma [3]

$$
\begin{aligned}
\left.S\left[x, y\left|\left[\begin{array}{ll}
1, & 0 \\
1, & 1
\end{array}\right]_{\mu}^{\lambda}\right|\left(\begin{array}{cc}
1, & 1 \\
1, & 1
\end{array}\right) \begin{array}{c}
1-\rho_{1} \mid\left(\begin{array}{ll}
1, & 1 \\
1, & 1
\end{array}\right) \\
0
\end{array} \mid\right]-\rho_{2} \mid\right] \\
=\frac{\Gamma(\lambda) \Gamma\left(\rho_{1}\right) \Gamma\left(\rho_{2}\right)}{\Gamma(\mu)} F_{1}\left[\lambda ; \rho_{1}, \rho_{2} ; \mu ;-x,-y\right],
\end{aligned}
$$

(for Appell function $F_{1}$, see $[1$, p. $224(6)]$ ) in (5), we have 


$$
\begin{aligned}
\sum_{n=0}^{\infty} \frac{(-1)^{n} \Gamma(\alpha-n \beta)}{n ! \Gamma(\alpha-n \beta-n)} F_{1}\left[\alpha-n \beta ; \rho_{1}, \rho_{2} ; \alpha-n-n \beta ; x, y\right] t^{n} \\
=\frac{(1+v)^{1-\alpha}}{1-\beta v}\left(1-\frac{x}{1+v}\right)^{-\rho_{1}}\left(1-\frac{y}{1+v}\right)^{-\rho_{2}} .
\end{aligned}
$$

In case we use the relation due to Erdélyi [1, Equation (3), p. 1216] and using the notation for hypergeometric functions of higher order and of two variables due to Chaundy [8] in (5), we get

$$
\begin{aligned}
\sum_{n=0}^{\infty} \frac{(-1)^{n} \Gamma(\alpha-n \beta)}{n ! \Gamma(\alpha-n-n \beta)} F\left[\begin{array}{c}
\alpha-n \beta ;-;-;-x,-y \\
\alpha-n-n \beta ; 1+\rho_{1} ; 1+\rho_{2}
\end{array}\right] t^{n} \\
=\frac{(1+v)^{1-\alpha} \Gamma\left(1+\rho_{1}\right) \Gamma\left(1+\rho_{2}\right)}{1-\beta v}\left(\frac{x}{1+v}\right)^{-\rho_{1} / 2}\left(\frac{y}{1+v}\right)^{-\rho_{2} / 2} \\
\cdot J_{\rho_{1}}\left(2\left(\frac{x}{1+v}\right)^{1 / 2}\right) J_{\rho_{2}}\left(2\left(\frac{y}{1+v}\right)^{1 / 2}\right) .
\end{aligned}
$$

In case $y=0$ in (8), it gives

$$
\sum_{n=0}^{\infty} \frac{(-1)^{n} \Gamma(\alpha-n \beta)}{n ! \Gamma(\alpha-n-n \beta)}{ }_{1} F_{2}\left(\alpha-n \beta ; \alpha-n-n \beta, 1+\rho_{1} ;-x\right) t^{n}
$$

$$
=\frac{\Gamma\left(1+\rho_{1}\right)(1+v)^{1-\alpha}}{1-\beta v}\left(\frac{x}{1+v}\right)^{-\rho_{1} / 2} J_{\rho_{1}}\left(2\left(\frac{x}{1+v}\right)^{1 / 2}\right) .
$$

We use Erdélyi's formula [1, (57), p. 220] in (5) to obtain

$$
\begin{gathered}
\sum_{n=0}^{\infty} \frac{(-1)^{n} \Gamma(\alpha-n \beta)}{n ! \Gamma(\alpha-n-n \beta)} F\left[\begin{array}{c}
\alpha-n \beta ; 1 / 2 ; 1 / 2 ;-x,-y \\
\alpha-n-n \beta ; 1 \pm \rho_{1} ; 1 \pm \rho_{2}
\end{array}\right] t^{n} \\
=\frac{(1+v)^{1-\alpha} \Gamma\left(1 \pm \rho_{1}\right) \Gamma\left(1 \pm \rho_{2}\right)}{(1-\beta v)} J_{\rho_{1}}\left(\left(\frac{x}{1+v}\right)^{1 / 2}\right) \\
\cdot J_{-\rho_{1}}\left(\left(\frac{x}{1+v}\right)^{1 / 2}\right) J_{\rho_{2}}\left(\left(\frac{y}{1+v}\right)^{1 / 2}\right) J_{-\rho_{2}}\left(\left(\frac{y}{1+v}\right)^{1 / 2}\right) .
\end{gathered}
$$

Next we use the formula due to Sharma [3]

$$
\left.\begin{array}{c}
\operatorname{Lt}_{x \rightarrow 0} S\left[x, y\left|\left[\begin{array}{cc}
m_{1}, & 0 \\
p_{1}, & q_{1}
\end{array}\right]_{1-b_{q_{1}}}^{1-a_{p_{1}}}\right|\left(\begin{array}{c}
n_{2}, m_{2} \\
p_{2}, q_{2}
\end{array}\right)_{d q_{2}}^{c_{p_{2}}}\left|\left(\begin{array}{c}
1,0 \\
0,1
\end{array}\right)_{0}^{-}\right|\right] \\
=G_{p_{2}+p_{1}, q_{2}+q_{1}}^{n_{2}, m_{2}+m_{1}}\left[\left.y\right|^{a_{1}, \cdots, a_{m_{1}}, c_{p_{2}}, a_{m_{1}+1}, \cdots, a_{p_{1}}}\right. \\
d_{q_{2}}, b_{q_{1}}
\end{array}\right]
$$


in (3); we have

$$
\begin{gathered}
\sum_{n=0}^{\infty} \frac{(-1)^{n}}{n !} G_{p_{1}+1, q_{1}+1}^{n_{1}, m_{1}+1}\left(x \mid \begin{array}{c}
1-\alpha+n \beta ; c_{p_{1}} \\
d_{q_{1}}, 1-\alpha+n+n \beta
\end{array}\right) t^{n} \\
=\frac{(1+v)^{1-\alpha}}{1-\beta v} G_{p_{1}, q_{1}}^{n_{1}, m_{1}}\left(\frac{x}{1+v} \mid \begin{array}{c}
c_{p_{1}} \\
d_{q_{1}}
\end{array}\right) .
\end{gathered}
$$

Also

$$
\begin{array}{r}
\sum_{n=0}^{\infty} \frac{(-1)^{n}}{n !} G_{p_{1}+1, q_{1}+1}^{n_{1}+1, m_{1}}\left(x \mid \begin{array}{c}
c_{p_{1}}, \alpha-n-n \beta \\
\alpha-n \beta, d_{q_{1}}
\end{array}\right) t^{n} \\
=\frac{(1+v)^{1-\alpha}}{1-\beta v} G_{p_{1, q_{1}}}^{n_{1, m_{1}}}\left(x(1+v) \mid \begin{array}{c}
c_{p_{1}} \\
d_{q_{1}}
\end{array}\right) .
\end{array}
$$

$\beta=-1$ and $\alpha=0$ in (12) reduces to a result due to Meijer [4, (33), p. 483].

$\beta=0, \alpha=1$ in (12) gives a result due to Meijer [4, (36), p. 485].

$\beta=0, \alpha=1$ in (13) gives a result due to Meijer [4, (37), p. 485].

$\beta=-1, \alpha=0$ in (13) reduces to a result due to Meijer [4, (46), p. 487].

If we use the formula $[1,(1), p .215]$ in (12) we get a result due to

Srivastava $[5,(9), \mathrm{p} .591]$. If we specialize the parameters of (12) we get the result due to Carlitz $[7,(8)$, p. 826].

\section{REFERENCES}

1. A. Erdélyi, Higher transcendental functions. Vol. I. The hypergeometric function, Legendre functions, McGraw-Hill, New York, 1953. MR 15, 419.

2. B. L. Sharma, On the generalized function of two variables. I, Ann. Soc. Sci. Bruxelles Sér. I 79 (1965), 26-40. MR 31 \#2425.

3. - On the generalized function of two variables. II, III (Communicated for publication).

4. C. S. Meijer, Expansion theorems for the G-function. II, Nederl. Akad. Weten sch. Proc. Ser. A 55 = Indag. Math. 14 (1952), 483-487. MR 14, 642.

5. H. M. Srivastava, Generating functions for Jacobi and Laguerre polynomials, Proc. Amer. Math. Soc. 23 (1969), 590-595. MR 40 \# 2935.

6. J. W. Brown, On zero type sets of Laguerre polynomials, Duke Math. J. 35 (1968), 821-823. MR $38 \# 2348$.

7. L. Carlitz, Some generating functions for Laguerre polynomials, Duke Math. J. 35 (1968), 825-827. MR 39 \# 1700.

8. T. W. Chaundy, Expansion of hypergeometric functions, Quart. J. Math. Oxford Ser. 13 (1942), 159-171. MR 4, 197. 